\title{
Comparison of abdominal compression devices in persons with abdominal paralysis due to spinal cord injury
}

\author{
Michaela de Groot ${ }^{1} \cdot$ Jennifer Swartz ${ }^{2} \cdot$ Jennifer Hastings $^{2}$
}

Received: 6 December 2018 / Revised: 6 March 2019 / Accepted: 17 March 2019

(c) International Spinal Cord Society 2019

\begin{abstract}
Study Design Single subject design with five subjects.

Objectives The objetive of this study is to compare the effectiveness and usability of alternative commercial abdominal compression garments with participants' usual medical binders.

Setting Private residences in Pierce and King Counties, WA, USA.

Methods Participants wore each garment for 5 days followed by a 2-day washout in personal binder. Week 1: Personal binder. Weeks 2 and 3: Randomly ordered test garments (tank, bodysuit). Physiologic measurements: blood pressure (SBP, DBP), blood oxygen saturation $\left(\mathrm{SaO}_{2}\right)$, forced expiratory volume in one second $\left(\mathrm{FEV}_{1}\right)$, and heart rate $(\mathrm{HR})$. Participants completed logs twice daily for 5 days per garment regarding ease of use, comfort, respiration, and appearance. We certify that all applicable institutional and governmental regulations concerning the ethical use of human volunteers were followed during the course of this research.

Results The use of a personal binder results in significant increases in SBP and FEV . Personal binders support FEV $_{1}$ significantly better than test garments. There is no difference in SBP between test garments and personal binders. There are no significant differences between $\mathrm{DBP}, \mathrm{SaO}_{2}$, or HR between participants' personal binders and no binder. Participants reported that neither tank nor bodysuit felt adequately supportive or easy to use.

Conclusions Abdominal compression improves respiratory function and supports SBP in individuals with chronic SCI. Further research is needed to guide the development of an easy-to-use and physiologically supportive abdominal compression garment.
\end{abstract}

\section{Introduction}

Spinal cord injuries (SCI) are classified according to the International Standards for the Neurological Classification of Spinal Cord Injury based on the severity of motor and sensory deficits and location of injury to the spinal cord. The extent of impairment depends on the severity of the injury to the spinal cord, ranging from incomplete injury with some motor or sensory function preserved to a complete injury resulting in total loss of function. Complete SCI affects nerves supplying muscles, resulting in a loss of

Jennifer Hastings

jhastings@ @ugetsound.edu

University of Puget Sound, Tacoma, WA, USA

2 University of Puget Sound, 1500 N Warner St. CMB 1030, 98416 Tacoma, WA, USA volitional contractions and resting muscle tone, which is problematic when those muscles are responsible for respiration and cardiovascular (CV) functions.

Cervical SCI impacts muscles controlling inhalation and exhalation, whereas thoracic and lumbar SCI affects abdominal muscles used for expiration. In cervical SCI, upper respiratory muscles adopt functions of respiration; however, abdominal muscles innervated by thoracic nerves are necessary for forced expiration, in order to execute a productive cough. Individuals with thoracic and lumbar SCI may be able to utilize abdominal muscles for respiration and resting tone to create a supportive abdominal wall, but abdominal muscle innervation is absent in patients with cervical and high thoracic SCI. Without abdominal wall support, intrathoracic pressure is drastically reduced, affecting cardiorespiratory systems. In supine, current literature shows no difference in pulmonary function between abdominal compression and no compression [1-3]. When seated upright, there is gravitational collapse and distension 
of the abdominal wall and diaphragm due to the injuryspecific lack of volitional motor control and resting tone within the abdomen resulting in reduced vital capacity, inspiratory and expiratory pressures, and lung and chest wall compliance [4]. Laxity in the abdominal wall can also result in difficulties with speech $[5,6]$ and decreased cough efficiency, which is critical for the ability to clear secretions from lungs $[4,7]$ to avoid respiratory infection. Acutely, most individuals with SCI employ the use of an abdominal binder, which mimics the intrathoracic pressure essential to $\mathrm{CV}$ and respiratory function. Despite the physiological benefits of abdominal compression, many individuals with chronic SCI discontinue the use of an abdominal binder after discharge from hospital or rehabilitation settings.

It is important to determine the effects of abdominal compression in a sitting position, as a majority of the SCI population uses a wheelchair; however, the literature is sparse. West et al. [8] investigated the effects of abdominal compression on respiratory mechanics during submaximal and maximal exercise in wheelchair rugby players with SCI. They showed abdominal compression resulted in increased vital capacity, whereas residual volume and functional residual capacity were reduced, and total lung capacity was relatively well preserved. In another study, West et al. [9] found an increase in vital capacity, expiratory flow, and inspiratory capacity when wearing an abdominal binder for non-athlete participants with low-cervical SCI. In addition, they found abdominal compression reduced residual volume as well as reduced functional residual capacity. A recent meta-analysis [2] showed patients exhibited significantly greater total lung capacity, forced vital capacity, and maximal expiratory pressure when wearing a binder. One study [3] found significant improvements in maximal expiratory pressure in a seated position with abdominal compression compared with those without; yet, there was no difference in maximal expiratory pressure in supine, supporting the use of compression when seated. Patients also reported feeling "greater ease in breathing" and coughing when wearing a binder while seated [3, 10]. Collectively, current research suggests abdominal compression may be most appropriate when seated to maintain respiratory parameters.

Few studies have investigated the effects of abdominal compression on $\mathrm{CV}$ responses within the general SCI population. A study [11] of wheelchair athletes with SCI showed no difference in heart rate (HR) or $\mathrm{VO}_{2}$ max during submaximal or maximal exercise testing, while using an abdominal binder. Huang et al. [1] found that SCI level does not significantly have an impact on whether compressive devices help with CV or pulmonary responses to postural change, although abdominal compression can help maintain blood pressure during a postural change and decrease orthostatic response. For patients with low-cervical SCI, abdominal compression can increase cardiac output by $28 \%$ and enhance diaphragmatic pressure production and improve left-ventricular function [9].

Research is limited regarding the experience of abdominal compression for individuals with chronic SCI. In a recent descriptive study investigating beliefs and barriers surrounding the use of abdominal binders, Hastings [12] found a majority of respondents believed the primary purpose of an abdominal binder is for postural support, while a secondary benefit is blood pressure support. Reported barriers to consistent abdominal binder use include skin breakdown, uncomfortable movement of the binder, and increased temperature due to the extra layer. These issues may contribute to the high rate of discontinued abdominal binder use following hospital discharge.

This study explores the physiologic benefit of abdominal compression in spinal cord injured individuals who regularly use a device and their response, both physiological and subjective, to alternative garments. Understanding factors that influence the daily use of abdominal compression from patients' perspectives will support future research in the design of more effective and patient-friendly devices.

\section{Methods}

This study followed a single subject design with participants acting as their own controls. This approach was selected to accommodate variability in terms of injury level, time since injury, age, sex, and type of personal abdominal binder used. Participants were recruited via convenience sampling of adaptive sports teams and support groups local to the research team in addition to the personal clinical client list of the primary researcher. Inclusion criteria included the following: (1) SCI at T6 or above (AIS A or B, to ensure complete abdominal paralysis), (2) understanding of written and spoken English, (3) daily user of an abdominal binder when seated upright in WC, and (4) able to don binder independently or with caregiver assistance. Exclusion criteria included the following: (1) current skin breakdown in region covered by the test garments, (2) unable to sit upright in WC for at least $6 \mathrm{~h}$ daily, and (3) require the use of mechanical ventilation when seated upright in WC. The institutional review board of the University of Puget Sound in Tacoma, WA, approved the ethics of the research protocol (protocol ID \#1617-089). We certify that all applicable institutional and governmental regulations concerning the ethical use of human volunteers were followed during the course of this research.

Data were collected over 3 periods of 1 week at a time. During each period, participants wore the compression garment for 5 days followed by a 2-day washout period in their personal abdominal binder. In week 1, participants 


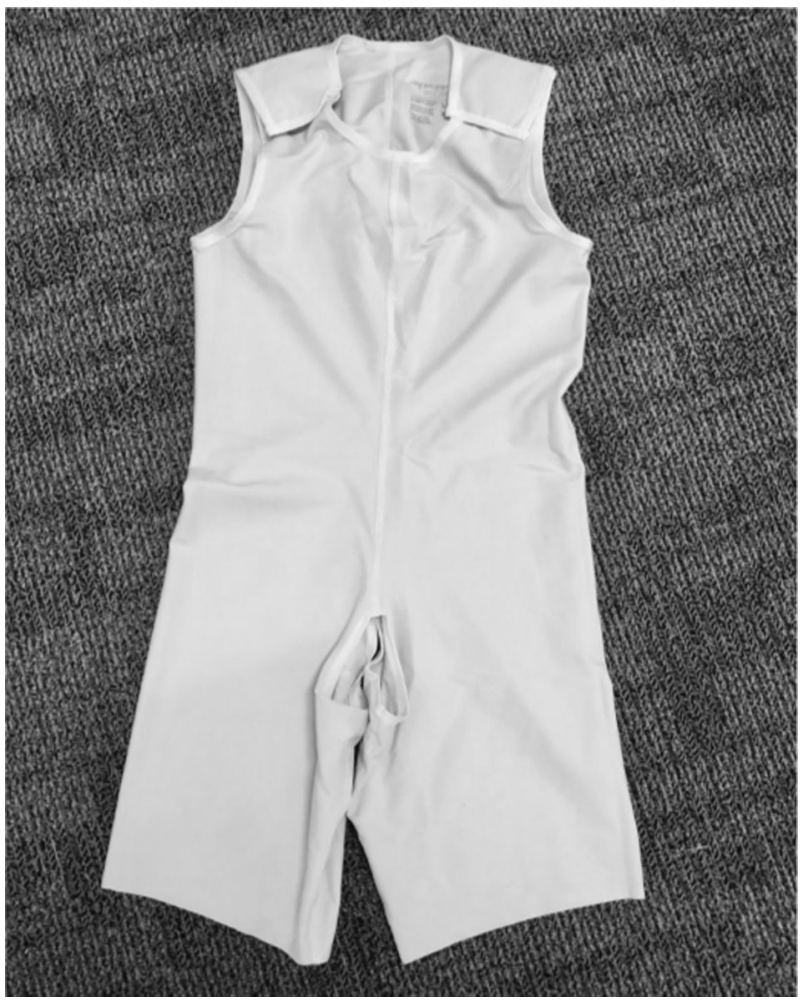

Fig. 1 Marena Recovery MB2 Sleeveless Compression Bodysuit. The Marena Group, Lawrenceville, GA, USA

wore their usual abdominal binder. In weeks 2 and 3, a test garment was worn in a randomized order determined using a random number order generator. The test garments are commercially available shapewear and chosen based on previous research findings that patients prefer an abdominal binder that is easy to don, lightweight, stays in place, and appears invisible under clothes [12]. The selected test garments were a bodysuit (Marena Recovery MB2 Sleeveless Compression Bodysuit, The Marena Group, Lawrenceville, GA, USA; Fig. 1) and a tank (Leo by Leonisa Firm Control Tank, Leonisa USA, Norcross, GA, USA; Fig. 2). The bodysuit was chosen to give a streamlined appearance under clothes and the tank was chosen to offer an easier donning and doffing experience. Garments were ordered according to the manufacturer's sizing recommendation based on each participant's waist, hip, and chest measurements.

Objective measures were taken on the first day of each data collection period by the research team in participants' homes. These visits enabled the research team to ensure proper fit of the test garment for each data collection period. At each visit, objective measures were taken (1) with the participant wearing their personal binder, (2) with no binder, following $5 \mathrm{~min}$ acclimatization, and (3) in the test garment, following $5 \mathrm{~min}$ acclimatization. All objective measures were collected with the participant in an upright seated position. Objective measures taken were systolic and

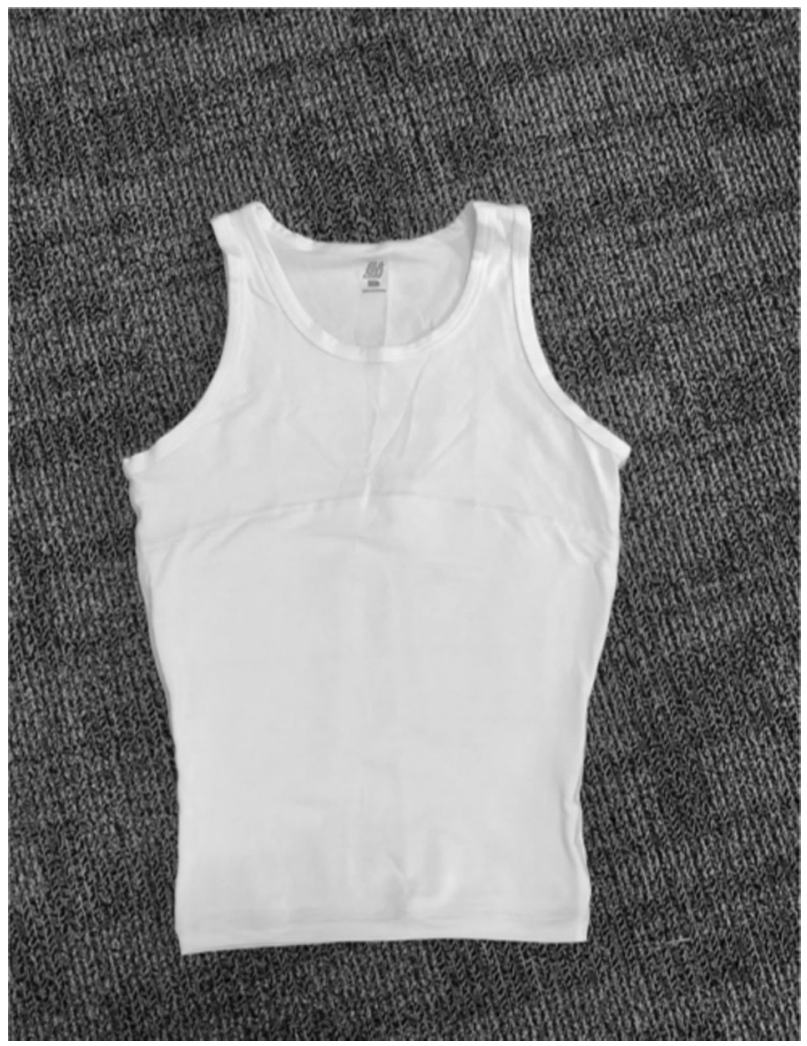

Fig. 2 Leo by Leonisa Firm Control Tank. Leonisa USA, Norcross, GA, USA

diastolic blood pressure (SBP, DBP), blood oxygen saturation $\left(\mathrm{SaO}_{2}\right), \mathrm{HR}$, and maximal forced exhalation volume in $1 \mathrm{~s}\left(\mathrm{FEV}_{1}\right)$. Blood pressure (SBP/DBP) was measured using an upper arm electronic BP cuff (model BP971-IT; Omron, Kyoto, Japan) on the participant's dominant side. Blood oxygen saturation $\left(\mathrm{SaO}_{2}\right)$ and $\mathrm{HR}$ were measured using a finger pulse oximeter (model SM165; Santa Medical, Tustin, CA, USA) on the same side as BP. Respiratory function $\left(\mathrm{FEV}_{1}\right)$ was assessed using a digital spirometer (model PF100; Microlife AG Swiss Corporation, Widnau, Switzerland). Each participant was permitted one practice attempt and three recorded attempts to achieve their maximal $\mathrm{FEV}_{1}$.

Beginning in the evening of the first day of each period, participants filled out twice-daily experience logs for 5 days per garment, for a total of nine entries per period. Experience logs included space for participants to record comments about each garment and visual analog scales (VAS) regarding comfort, ease of use, appearance, and respiration. Experience logs were collected by the research team at the end of each period.

$\mathrm{CV}$ and respiratory function data were analyzed in SPSS using paired $t$-tests of differences to compare participants' usual binders with no binder, usual binders with test garments, and test garments with no binder. Test garments 
Table 1 Participants' characteristics and profile

\begin{tabular}{lllllll}
\hline Subject & Sex & Age (years) & Level of injury & BMI & Years post-SCI & Years wearing binder \\
\hline 1 & M & 37 & C3-4 AIS A & 26 & 13 & 13 \\
2 & M & 28 & C5 AIS A & 32 & 1 & 1 \\
3 & M & 65 & C5-6 AIS A & 23 & 47 & 15 \\
4 & M & 47 & T4 AIS A & 26 & 21 & 9 \\
5 & F & 36 & C4-5 AIS A & 21 & 17 & 17 \\
Mean & & 42.6 & & 25.5 & 19.8 & 11 \\
Median & & 37 & C5 & 26 & 17 & 13 \\
SD & & 14.22 & & 4.16 & 16.95 & 6.32 \\
Range & $28-65$ & C3-T4 & $21-32$ & $1-47$ Years & $1-17$ Years \\
\hline
\end{tabular}

were aggregated into a single category for the purposes of statistical analysis. Subjective data were analyzed across participants and coded for themes independently by researchers JS and $\mathrm{MD}$, with $\mathrm{JH}$ acting as mediator for any disputes.

\section{Results}

Seven individuals were screened and five enrolled during the recruitment period. All participants completed an initial screening interview to determine eligibility and provided written consent before proceeding. Table 1 summarizes participants' demographic information.

Participants wore their own abdominal binders during the initial week of data collection and during all washout periods. Although binders vary by manufacturer, four of five participants use some form of three-panel elastic binder that overlaps across the anterior abdomen to close with Velcro, with personal preference determining right- or left-sided closure (similar to what is shown in Fig. 3). Participant 4 uses a seamless neoprene binder.

Outcome measures were collected on Participants 1-4 in all garments. The research team was unable to collect objective measures of one subject (Participant 5) in the tank or bodysuit because of her inability to don either garment. This subject has significantly limited shoulder abduction and flexion range of motion (ROM) that precluded her from donning the tank. Although garments were ordered based on participants' unique measurements, the research team was unable to find a bodysuit that was appropriately compressive for this subject without being unacceptably small in other dimensions.

Use of participants' usual medical binders resulted in significant increases in SBP $(p=0.009)$ compared with no binder. We found a moderate to large effect size using Cohen's $d(d=1.11)$, suggesting strong practical importance. Use of participants' usual medical binders resulted in significant increases in $\operatorname{FEV}_{1}(p=0.000)$ compared with no binder. We found a very large effect size using Cohen's $d$

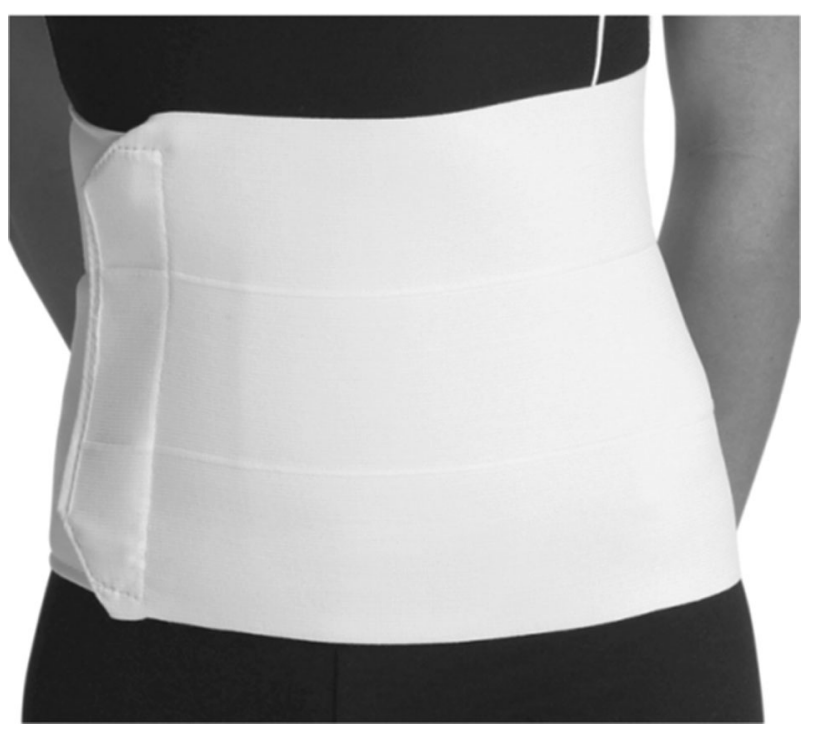

Fig. 3 Typical paneled elastic abdominal binder. ProCare Premium Panel Elastic Binder. DJO LLC, Vista, CA, USA

$(d=2.31)$, suggesting strong practical importance. Participants' usual medical binders support $\mathrm{FEV}_{1}$ significantly better than the test garments $(p=0.034)$. We found a moderate to large effect size using Cohen's $d(d=1.35)$, suggesting strong practical importance. Statistically, test binders were not different than participants' usual medical binders for SBP $(p=0.624)$; however, this may reflect autonomic dysreflexia (AD) caused by the bodysuit. Table 2 summarizes mean $\mathrm{FEV}_{1}$ for each participant. Table 3 summarizes SBP and DBP for each participant.

Table 2 Mean $\mathrm{FEV}_{1}$ (L) for each participant by binder type

\begin{tabular}{llllll}
\hline Subject & 1 & 2 & 3 & 4 & 5 \\
\hline No binder & 1.91 & 2.97 & 2.08 & 3.13 & 0.89 \\
Own binder & 2.24 & 3.18 & 2.33 & 3.26 & 1.13 \\
Tank & 2.2 & 3.02 & 2.15 & 3.01 & - \\
Bodysuit & 2.2 & 2.46 & 2.2 & 3.19 & - \\
\hline
\end{tabular}


Table 3 Mean systolic and diastolic blood pressure $(\mathrm{mm} \mathrm{Hg})$ for each participant by binder type

\begin{tabular}{lllllll}
\hline Subject & & 1 & 2 & 3 & 4 & 5 \\
\hline No binder & SBP & 89 & 107 & 94 & 112 & 104 \\
& DBP & 63 & 87 & 70 & 78 & 81 \\
Own binder & SBP & 93 & 114 & 109 & 111 & 117 \\
& DBP & 70 & 83 & 77 & 75 & 89 \\
Tank & SBP & 94 & 99 & 100 & 113 & - \\
\multirow{3}{*}{ Bodysuit } & DBP & 68 & 66 & 80 & 73 & - \\
& SBP & 97 & 137 & 212 & 123 & - \\
& DBP & 70 & 92 & 82 & 87 & - \\
\hline
\end{tabular}

Participants completed experience logs for each data collection period and were encouraged to make notes about their impressions of each binder type. Regarding comfort, participants reported finding the tank "warmer" than their usual binder, whereas others said they felt it was "not as stable as [their] own binder." Others stated they were "impressed that it stays in place during an active day," and that they "really like that it doesn't cut or pinch." Participants found the bodysuit unacceptably uncomfortable: "my stomach doesn't feel bound," "isn't compressing where it's supposed to," "rotates around my genitals," and "shoulder straps scrunch my posture." Regarding appearance, participants reported liking the "seamless appearance" and "smooth chest-to-belly transition" of the tank, although one participant reported a "persistent 'muffin top' just above the belt line." Participants stated the bodysuit was "not very masculine" and "not something [they] would use on [their] own." No participant offered comments specific to respiration, although several reported finding that neither the tank nor bodysuit seemed as compressive or stable as their own binder. Participants reported ease of use complaints with the tank and bodysuit, finding both difficult to don and doff, and in the case of the bodysuit, some required an additional caregiver for assistance. VAS data for Participant 1 regarding ease of breathing and trunk stability are shown in Figs. 4 and 5, respectively.

One of five participants (Participant 1) completed VAS data for all three data collection periods of the study, whereas four of five gave partial VAS data before dropping out (dropout rate $=80 \%$ ). Reasons for dropout were specific to the test garments. For the tank, dropout occurred due to difficulty or outright inability to don the garment due to upper extremity ROM limitations, incompatibility of manufacturer's garment sizing with participants' measurements, and dissatisfaction with the appearance of the tank under clothing. For the bodysuit, dropout occurred due to unacceptable discomfort while wearing the garment, particularly around the shoulders, concern about movement of the garment affecting insensate genitals, and inability for those participants typically independent in dressing to don the garment without assistance. Only two participants were willing, or able, to wear the bodysuit beyond the collection of baseline physiologic data.

\section{Discussion}

This study has shown positive, significant evidence that abdominal compression improves respiratory function and supports SBP in individuals with chronic SCI. Our results suggest that recommendation of long-term abdominal compression may be appropriate for all patients with abdominal paralysis due to chronic SCI. Importantly, although test garments provided similar SBP support as usual medical binders, this finding may have been skewed by mild AD induced by the suit for one participant (Participant 3). It is difficult to say definitively whether Participant 3's increased SBP, while wearing the suit was truly $\mathrm{AD}$ or simply age-related hypertension. Donning the suit caused frustration and even anger in other participants, which may have resulted in elevated blood pressure. Neither Participant 3 nor any other participants reported usual symptoms for $\mathrm{AD}$, while wearing the suit, and none sustained any injury from the suit.

We found barriers to the adoption and use of certain styles of abdominal compression, which agree with previous research in this area [12]. It is clear that regardless of cardiopulmonary support, a garment that is neither comfortable nor user-friendly will not be readily adopted by this population. It is important to consider how each of this study's participants, who vary widely in both years since injury and years using an abdominal binder, came to be daily users of abdominal compression. Some have been using a binder since their initial injury and subsequent hospitalization, whereas others went many years between wearing a binder during their inpatient stay and resuming binder use later on.

This study has several limitations. First, objective measures from all participants were aggregated by garment type for analysis. This means that participants' usual medical binders were treated as categorically identical, despite one using a neoprene binder that differs in construction and material from the three-panel elastic binders worn by the rest of the participants. In addition, data from both the tank and bodysuit were aggregated into a single "test garment" category for statistical analysis. The decision to aggregate groups of garments for analysis comes at the expense of discerning differences between specific garments. It is possible that either the tank or bodysuit provided greater physiologic support than the other and it is likely to be that the elevated SBP measured in the bodysuit skewed our result. 
Fig. 4 VAS data for Participant 1 in response to the prompt, "Rate how easy it is to breathe in this garment."
Fig. 5 VAS data for Participant 1 in response to the prompt, "Consider your trunk stability: How stable do you feel while wearing the garment?"
Participant 1: Ease of Breathing in Personal Binder vs Tank vs Bodysuit
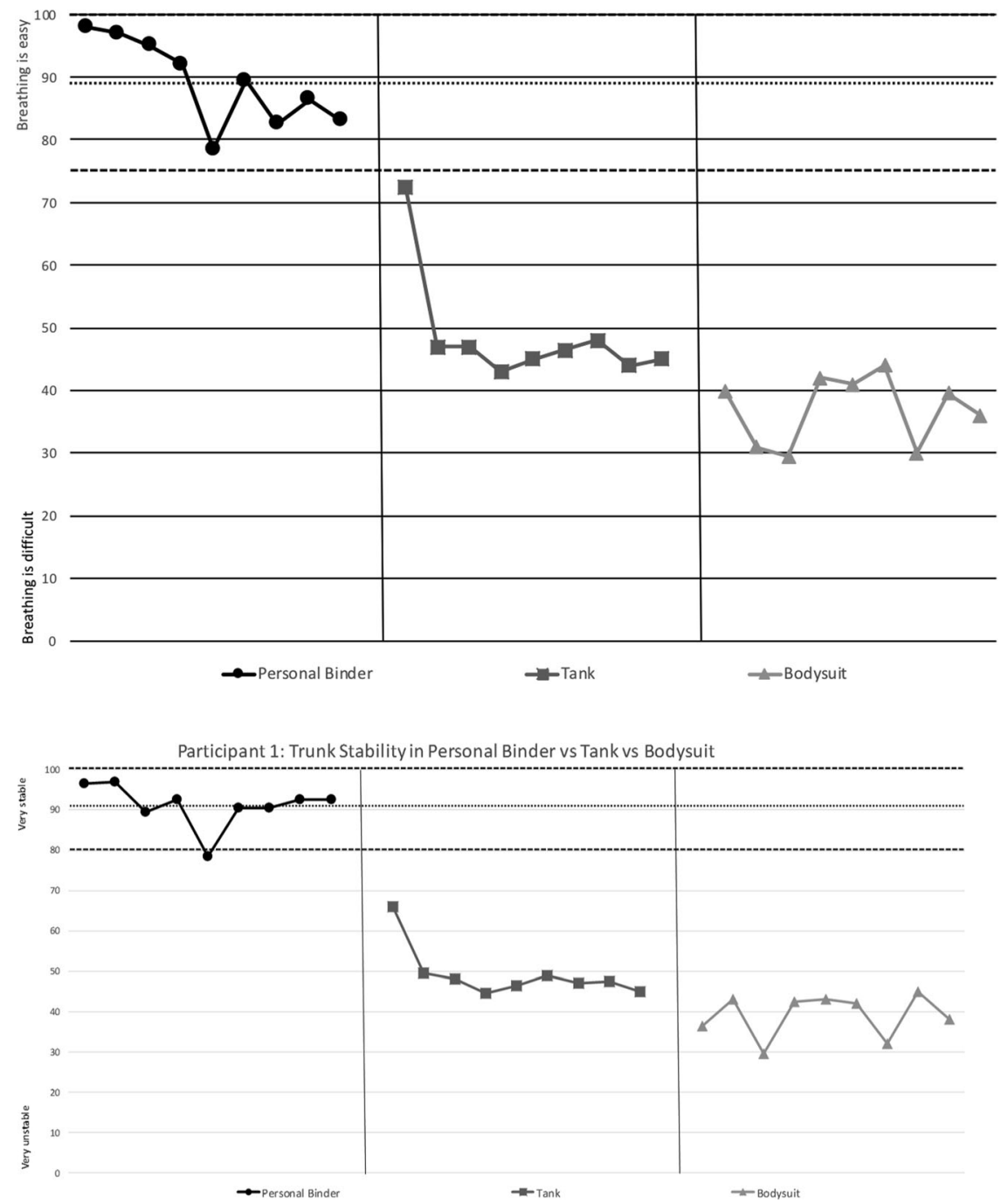

A second limitation of this study is the novelty of the test garments and the relatively brief data collection period. Participants wore their test garments for only 5 days before switching back to their usual binders. That this study asked participants to temporarily replace something they have understood confers vital physiologic support with a novel garment that is untested in providing physiologic support may have contributed to early dropout and negative perceptions, comments, and low VAS scores. The brief duration of each test period may have been insufficient to allow participants to become fully accustomed to their test garments, thus artificially capturing negative "first impression"-type opinions. There is some evidence supporting this hypothesis: in the weeks following data collection, two participants reached out to the research team for purchasing information on the tank so that they could order additional garments. These participants reported that they'd come to like the way the tank looks under clothing and found the support to be similar to their usual binders, comments that contradict these participants' earlier entries in their experience logs. Unfortunately, it remains true that there are barriers to the use of long-term compression due to previously described issues with the current devices on the market designed for this purpose. Although the commercial shapewear industry offers some products that may work for this population, it is difficult to size garments off the shelf using manufacturer's size guides due to the combination of skeletal muscle atrophy and stomach distention that occurs in the chronic SCI population.

The results of this study add to the literature for health professionals to utilize in educating patients. It is vital that all members of the rehabilitation team-from physiatrists to nurses, to occupational and physical therapists-be wellversed in the benefits of long-term abdominal compression 
for this population, so that all rehabilitation team members are able to provide consistent patient education and recommendations to continue abdominal compression post discharge. Further research is needed to see whether adherence to the long-term use of abdominal compression would be different if individuals were transitioned from the use of the usual medical binders to the use of garment-style compression upon discharge to home from their inpatient rehabilitation, before significant abdominal distention has occurred.

We conclude that abdominal compression is an effective tool for maintaining SBP and supporting forced expiration, both critically important physiologic functions for individuals with SCI. Therefore, it is recommended that all individuals with SCI with abdominal paralysis use longterm abdominal compression. It is evident from this study that what is needed next is an expansion of the abdominal compression garment market for individuals with abdominal paralysis, resulting in choices of garments that are physiologically supportive and easy to use.

\section{Data archiving}

The datasets generated and analyzed during the current study are available from the corresponding author on reasonable request.

Acknowledgements We acknowledge the University Enrichment Committee and School of Physical Therapy at University of Puget Sound in Tacoma, WA, for its support of this project.

\section{Compliance with ethical standards}

Conflict of interest The authors declare that they have no conflict of interest.

Publisher's note: Springer Nature remains neutral with regard to jurisdictional claims in published maps and institutional affiliations.

\section{References}

1. Huang CT, McEachran AB, DeVivo MJ. Fine PR cardiopulmonary response in spinal cord injury patients: effect of pneumatic compressive devices. Arch Phys Med Rehabil. 1983;64:101-6.

2. Wadsworth BM, Haines TP, Cornwell PL, Paratz JD. Abdominal binder use in people with spinal cord injuries: a systematic review and meta-analysis. Spinal Cord. 2009;47:274.

3. Boaventura CM, Gastaldi AC, Silveira JM, Santos PR, Guimaaes RC, De Lima LC. Effect of an abdominal binder on the efficacy of respiratory muscles in seated and supine tetraplegic patients. Physiotherapy. 2003;89:290-5.

4. Brown R, DiMarco AF, Hoit JD, Garshick E. Respiratory dysfunction and management in spinal cord injury. Respir Care. 2006;51:853-70.

5. Cornwell P, Ward E, Lim Y, Wadsworth B. Impact of an abdominal binder on speech outcomes in people with tetraplegic spinal cord injury: perceptual and acoustic measures. Top Spinal Cord Inj Rehabil. 2014;20:48-57.

6. Wadsworth BM, Haines TP, Cornwell PL, Rodwell LT, Paratz JD. Abdominal binder improves lung volumes and voice in people with tetraplegic spinal cord injury. Arch Phys Med Rehabil. 2012;93:2189-97.

7. Estenne M, Van Muylem A, Gorini M, Kinnear W, Heilporn A, De Troyer A. Effects of abdominal strapping on forced expiration in tetraplegic patients. Am J Respir Crit Care Med. 1998;157:95-8.

8. West CR, Goosey-Tolfrey VL, Campbell IG, Romer LM. Effect of abdominal binding on respiratory mechanics during exercise in athletes with cervical spinal cord injury. J Appl Physiol. 2014;117:36-45.

9. West CR, Campbell IG, Shave RE, Romer LM. Effects of abdominal binding on cardiorespiratory function in cervical spinal cord injury. Respir Physiol Neurobiol. 2012;180:275-82.

10. Hart N, Laffont I, de La Sota AP, Lejaille M, Macadou G, Polkey MI, et al. Respiratory effects of combined truncal and abdominal support in patients with spinal cord injury. Arch Phys Med Rehabil. 2005;86:1447-51.

11. Kerk JK, Clifford PS, Snyder AC, Prieto TE, O'Hagan KP, Schot $\mathrm{PK}$, et al. Effect of an abdominal binder during wheelchair exercise. Med Sci Sports Exerc. 1995;27:913-9.

12. Hastings $J$. What are the barriers to use of abdominal binders in persons with abdominal paralysis due to spinal cord injury? Poster presented at: The 4th ISCoS and ASIA Joint Scientific Meeting 2015; Montreal, QC. 